\title{
A Data Fusion Methodology for Wireless Sensor Systems
}

\author{
J.I.-Z. Chen, Y.-N. Chung
}

\author{
Joy Iong-Zong Chen \\ Department of Electrical Engineering \\ Dayeh University \\ Changhua 51505,Taiwan (ROC) \\ E-mail: jchen@mail.dyu.edu.tw \\ Yi-Nung Chung \\ Department of Electrical Engineering \\ National Chunghua University of Education \\ Changhua 51505, Taiwan (ROC) \\ E-mail: yichung@ncue.dyu.edu.tw
}

\begin{abstract}
An efficient DFA (data fusion algorithm) plays an important role in tracking for moving objects over WSS (wireless sensor system) deployments in order to track the objects accurately. Accuracy in object tracking is mainly dominated by the prediction for those moving targets by filtering and refining the results from wireless mobile sensors deployed in WSS environment. A DFA based on CHHN (competitive Hopfield neural network) technique for obtaining the relationship between measurements results from wireless mobile sensors and estimation of existing tracks over WSS (wireless sensor system) is proposed in this paper. Embedded within the CHNN is also a competitive learning mechanism which creatively removes the dilemma of occasional irrational solutions in traditional HNN (Hopfield neural networks). In this research, except the proposed approach is established with CHNN, the methodology of data fusion over WSS is guaranteed to converge into a stable state when performing a data association. In words, the CHNN-based DFA is combined with wireless mobile sensors in a WSS environment to demonstrate the target tracking capabilities. Computer simulation results illustrate that the new methodology of data fusion based on CHNN is not only successfully able to solve the data association problems addressed over WSS environments, but the specified simulated targets can also be tracked without large scale missing.

Keywords: CHNN (competitive Hopfield neural network), DFA (data fusion algorithm), mobile sensors, WSN (wireless sensor network).
\end{abstract}

\section{Introduction}

Mobile-sensor data association tracking is generally an essential technique for WSS (wireless sensor system) surveillance systems employing one or more sensors, which may be deployed as stationary or maneuverable, together with computer subsystems. The main objective of a data association tracking algorithm is to partition sensor data into sets of observations produced by the same target; the other object to avoid the coupling effect existing between the mobile sensors for the same target. Once tracks are formed and confirmed, it not merely the number of mobile sensors that can be estimated and quantified; rather, the information gathered by the tracking algorithm can also be associated and fused. It is known that the role of multi-sensor data association in WSS environments involves acquiring, processing and combining data $[1,2]$ coming from different sources, including sensors and databases, into a more precise set of data. Thus, 
data association consists of the three aforementioned steps. A DFA (data fusion algorithm) is the most important technique for maintaining tracking procedures. Mobile-sensor tracking with a DFA is a prerequisite step for mobile-sensor surveillance systems in WSS deployments. Once tracks are formed and confirmed, the number of targets can be estimated and information, such as the target position and velocity, computed for each track [3]. In the literature, several DFAs for MTT (multiple target tracking) have been proposed and discussed. It is well known that the JPDA (joint probabilistic data association) DFA technique, discussed in References [4-8] focusing mainly on MTT deployment, is appropriate for a high false-target density environment. However, these techniques for solving MTT problems may cause some unreliability (latency) because in a nearest-neighbor or all-neighbors-based environment, the relationships between sensor measurements and existing target tracks are usually considered independently [9]. Thus, currently a traditional HNN (Hopfield neural network), which incorporates weighted objective costs and constraints into an overall energy function, is employed to combine with the neural network approaches for achieving good tracking results. [10] Then, through minimizing the overall energy function, superior performance results can be obtained. [11] The CHNN (competitive Hopfield neural network) algorithm has been applied in image processing. [12] Moreover, Soujeri and Bilgekul [13] adopted a conventional HNN to solve the problems of multiuser reception for asynchronous MC-CDMA (multi-carrier coded-division multiple-access) systems in multipath fading channels. Since this approach is defective in that the weighting values are very difficult to properly determine, frequently the solution obtains an irrational result, as reported by Zhou. [14] Thereafter, conventional HNN schemes for tracking maneuvering or non-maneuvering targets with mobile sensors over WSS deployments are very sparse. However, Wang et al. [15], recently, combined an HNN with a genetic algorithm, designated as HNN-GA, for proposing a mobile agent-based strategy utilizing a low network load and cooperation of mobile agents, to dynamically optimize the combination of nodes and deploy tasks among nodes. Based on HNN, the selection method investigated by Liang et al. [16], in which the sensor node having the lowest cost and satisfying the distance requirement of a MIMO (multi-input multi-output) system, is selected to function as the best transmitting and receiving antenna in WSN environments. By extending the idea expressed in [17], Wang et al. [18] proposed a new dynamic sensor node selection strategy to implement global searching to reduce the search space of a GA and ensure the validity of each chromosome in WSN applications. Deploying with mobile sensors in WSS environment is increasingly becoming integral to targets tracking, mainly due to its convenient deployment, small size, real-time characteristic, and flexibility to support integrated applications. The technique of WSS is applied to the traffic monitoring and control in [19] by the authors Semertzidis et al. The information is fused and used to provide real-time analytical. However, it is necessary for the important issue of energy supply to the operation of sensor nodes over WSS. Yen et al. in [20] proposed CLD (controlled layer deployment) protocol to guarantee coverage and energy efficiency for sensor nodes deployed in a WSS. In order to promote the operation efficiency of the data fusion for the sensor nodes deployed in WSS environment. Multi-layer clustering routing algorithm is presented by Liu et al. in [21] where the WSS techniques is developed to track the moving vehicles, and the new scheme efficiently overcomes the hot spot problem in WSS environment. Furthermore, the authors, Shi et al., propose a structure that represents the sensor communications with the fusion centre, obtain the optimal estimation algorithm at the fusion centre, and provide a theoretical closed-form for the steady-state error covariance matrix which has low energy consumption and guarantees a desired level of estimation quality at the fusion centre. [22] However, to apply the advantages of the HNN technique in this research the improved CHNN method, which can by artfully arranging the updating function and the cost measurement properly eliminate the aforementioned dilemma, is adopted for solving target tracking with mobile sensors in WSS deployments. The CHNN is an improved HNN wherein a 
cooperative decision is made on the basis of the simultaneous input of a community of neurons. Each neuron receives information from other neurons and also conveys information to others $[11,14]$. With this collective information, each neuron moves to a stable stage with the lowest value of a predefined energy function. With such a result, the operation of association between mobile sensor measurements and existing tracks can be obtained under global optimal consideration, which in turn can increase the accuracy of mobile sensor tracking systems. Furthermore, due to the embedded competitive updating scheme, the CHNN concept has the capability of removing the burden of weight setting. It has also been demonstrated that the network is guaranteed to converge into a stable and rational stage during evolution. [13] Thus, the dilemma of lapsing into irrational solutions such as those in traditional HNNs can be eliminated.

To the best of the author knowledge, the proposed mean is an innovative idea for discussion when exploring the field of WSNs. The aim of this study is to enhance system performance by means of a DFA for WSS environments, the focus being on a CHNN to obtain global matching between mobile sensor measurements and existing tracks. As illustrated in Fig.1, moving targets tracked by sensor nodes are considered for improving the tracking deployment for mobile-sensor data association with assumed targets. Moreover, in an environment where the dense targets are spread out randomly, some targets can be very close to each other. The measurements produced by these close targets can confuse the data association computation algorithm and result in inaccurate relations hips. Consequently, the approach for solving the data association problem should be considered globally. The reminder of this report is organized as follows. In Section II the problem formulation includes the gating technique, and the basic concept of the HNN is illustrated; developing a DFA based on the CHNN scheme is presented in Section III; the maneuvering compensator algorithm is explicated in Section IV; Section V reports the simulation results for the proposed algorithm; and Section VI both concludes the report and offers recommendations for further research.

\section{Problem Formulation}

In this sub-section to obtain the information from the mobile sensor belonging to all of the sensors, without loss of generality, the type of sensing methods is considered to be completely established. It is also assumed that the synchronization between various sensor levels under circumstances of multiple levels of data association is finished. Several synchronization methods can be adopted for this procedure [18]. Moreover, since this study focuses on data association for tracking targets within WSN environments, several issues are reasonably ignored, e.g., the impact of sensing accuracy on the sensing range and node densities of a sensor, as well as the data fusion overhead and sensing lifetime of a network. Thus, a dynamic model with a discrete model set for a multi-sensor tracking algorithm for a mobile sensor can be formulated.

\subsection{Gating Technique}

According to the mobile sensor tracking situation, the model of a moving target can be defined in variable state equations, given as [23]

$$
X(k+1)=F(k) X(k)+G(k) W(k)+U(k)
$$

and

$$
Y(k)=H(k) X(k)+V(k)
$$

where $X(k)$ denotes the state vector of the target, $Y(k)$, the measurement vector of the target, $F(k)$ and $G(k)$, the transition matrix and the noise-gain matrix of the target, respectively; $W(k)$, 
is the system noise associated with the target, assumed to be normally distributed with a zero mean and variance $Q(k)$. Moreover, $U(k)$ represents the forced input; $V(k)$, the measurement error associated with the target, assumed to be normally distributed with zero mean and variance, $R(k)$, uncorrelated with $W(k)$; and $H(k)$ the measurement matrix of the target. The initial state of the target is considered to be a Gaussian pdf (probability density function) with a known mean vector, $\widehat{X}(0 \mid 0)$, and a covariance matrix, $P(0 \mid 0)$. A large number of close measurements observed from the mobile sensors in an actual tracking situation are provided to determine the trajectory estimates for any target that might be presented. It is difficult to precisely determine which target corresponds to which in such closely spaced measurements. i.e., the data association problem in mobile sensor tracking or associating the measurement vector $Y(k)$ to the existing track model for each step, $k$, must first be solved. Additionally, consider an environment wherein a dense target is completely deployed. Here, determining the gating size is the obligatory first step in solving the problem of associating observations with tracks. Additional logic is required when an observation or multiple observations fall within the gates of multiple target tracks or a single track. A typical situation for a gate diagram consisting of three target tracks $T 1, T 2$ and $T 3$ is illustrated in Fig. 2. In this figure there are three targets, but eight measurements are assumed to have been obtained. First, the gating technique is applied to eliminate the less probable measurements such as $M_{6}$ to $M_{8}$. A CHNN algorithm is applied to complete the associative relationship between the remaining measurements and the tracked targets. Then, the association of the measurements of the remaining $M_{1}, M_{2}, \cdots, M_{5}$, with the respective targets should be determined.

\subsection{Two-dimensional Binary Neural Network-HNN}

Consider that a network consists of $n \times m$ mutually interconnected neurons. In this research, the HNN specifically adopted to search for the algorithm belongs to a two-dimensional binary neural network wherein the total input-to-neuron, indexed by $(g, i)$, i.e., $\Lambda_{g, i}$, at time $k$, can be calculated as [15]

$$
\Lambda_{g, i}=\sum_{h=1}^{n} \sum_{j=1}^{m} T_{g, j ; h, j} \cdot V_{h, j}+I_{g, i}
$$

where $I_{g, i}$ denotes a bias input, $T_{g, j ; h, j}$, the interconnection intensity between neuron $(g, i)$ and neuron $(h, j) ; V_{g, i}$, a binary state of the $(g, i)$ th neuron. Moreover, the state of $V_{g, i}$ is determined by the signum function

$$
V_{g, i}= \begin{cases}1, & \Lambda>0 \\ 0, & \text { otherwise }\end{cases}
$$

Thus, the expression in 1 explicitly describing the HNN dynamics operating in this network receives weighted inputs $T_{g, j ; h, j} \cdot V_{h, j}$ from each neuron, e.g., $(h, j)$, and a bias input $(g, i)$ from outside. Therefore, one neuron at a time is updated while the rest remain stationary after a random initialization of each neuron with binary values. This method for neuron updating has been shown to decrease the Lyapunov function of a two-dimensional Hopfield network given by

$$
E=-\sum_{g=1}^{n} \sum_{h=1}^{n} \sum_{i=1}^{m} \sum_{j=1}^{m} T_{g, j ; h, j} V_{g, i} V_{h, j}-2 \sum_{g=1}^{n} \sum_{i=1}^{m} I_{g, i} V_{g, i}
$$

where $V_{g, i}$ has the constraint defined in (4). 


\section{Joint Data Association in a Competitive Neural Network}

In this subsection the CHNN algorithm is applied to the potential-target measurements to formulate a solution for data association problems. The CHNN concept is applied to the data association technique presented here. By means of a network for joint data association, the state of $V_{g, i}$ indicates an associative status between the $g-t h$ mobile sensor measurement and the $i-t h$ target, expressed as

$$
V_{g, i}= \begin{cases}0, & \text { data noassociated } \\ 1, & \text { data associated }\end{cases}
$$

Then the objective function used for obtaining measurements and mobile sensor targets association with the best decision is given by

$$
E=\alpha \sum_{g=1}^{n} \sum_{i=1}^{m} d_{g, i} V_{g, i}+\beta \sum_{g=1}^{n} \sum_{h=1}^{n} \sum_{i=1}^{m} \sum_{j=1}^{m} V_{g, i} V_{h, j} \delta_{g, h}+\gamma \sum_{i=1}^{m}\left(\sum_{g=1}^{n} V_{g, i}-1\right)^{2}
$$

where $d_{g, i}$ is the distance between the $i-t h$ true target and the $g-t h$ measurement. The factor $d_{g, i}$ here needs a special design for achieving the task of data association as described subsequently. In Eq. 7 the first term is the sum of the distances between the associated measurements and the mobile sensor targets. A situation wherein none of the newly obtained measurements is appropriate for certain specific targets in the processing of data association is unavoidably vexing. Concurrently, the previous target information should be kept and chosen as the next item of information in such cases. To achieve this end, the $m$ target data is included as part of the measurement. Assume that there are $m$ targets and $n-m$ newly obtained mobile sensor measurements and that the $m$ targets are arranged in front of the $n-m$ measurements to obtain a total of $m$ measurements. Where $g=i$, the distance $d_{g, i}$ is defined as $d_{g, i}=r$ where $r$ is the radius of the gate, according to the aforementioned arrangements. Hence, if measurements are distributed inside the gate, then one should be chosen. However, if no measurements are there, then the target itself should be chosen. Another constraint is that if $g \neq i$ and $1 \leq g \leq m$, then $d_{g, i}=\infty$. This constraint prevents one target from choosing another as its measurement. Thus, after the aforementioned calculations, the distance $d_{g, i}$ can be determined as

$$
d_{g, i}= \begin{cases}r, & g=i \\ \infty, & g \neq i \text { and } 1 \leq g \leq m \\ \sqrt{\left[\tau^{T}(k) S(k)^{-1} \tau(k)\right]}, & g \neq i \text { and } g>m\end{cases}
$$

where $S(k)$ is the covariance matrix of the innovation $\tau(k)$, the superscript denotes the transportation of a matrix, and $\tau(k)=Y(k)-H \widehat{X}(k \mid k-1)$. Moreover, in 5 the second term attempts to guarantee that each measurement can be associated with only one target. Moreover, the third term forces the condition wherein each target has one, and only one, associated measurement and the three terms $\alpha, \beta$, and $\gamma$ specify important constant factors. Since these factors are highly dependent on the number of targets, the target-measurement distances and the radius of the gate, it is very difficult to determine the appropriate values for them. For this reason, irrational solutions have periodically been reported for the use of traditional Hopfield approaches $[10,14]$ when the weighting factors have not been properly determined. However, to reduce the difficulty in determining the values of the weighting factors, a CWTA (competitive winner-take-all) updating is proposed and adopted as

$$
V_{g, i}= \begin{cases}1, & \Lambda_{g, i}=\max \left\{U_{1, i} \cdots \cdots U_{n, i}\right\} \\ 0, & \text { otherwise }\end{cases}
$$


With this modified updating rule, the rigid constraint that each target should be associated with one and only one measurement is automatically embedded inside the network evolution results. As such, the third term can naturally be removed from the objective function. Thus, the objective function is simplified and obtained as

$$
E=\alpha \sum_{g=1}^{n} \sum_{i=1}^{m} d_{g, i} V_{g, i}+\beta \sum_{g=1}^{n} \sum_{h=1}^{n} \sum_{i=1}^{m} \sum_{j=1}^{m} V_{g, i} V_{h, j} \delta_{g, h}
$$

To avoid being bounded by irrational solutions, a reasonable method for the network described below is adopted. I.e., once the CWTA updating has been applied, it is noteworthy that if $\alpha$ is set as a unit, $\beta$ can be easily considered greater than the radius of gate $r$, a relatively constant value. By comparing the resultant objective function with the Lyapunov function of the twodimensional Hopfield network in 5, the parameters of the interconnection intensity and the bias input can be obtained as

$$
I_{g, i}=-\frac{\alpha \cdot d_{g, i}}{2}
$$

and

$$
T_{g, i ; h, j}=-\beta \delta_{g, h}
$$

respectively. It can be clearly to seen that the CHNN is not fully interconnected from, 11 and 12. However, the CHNN is locally connected instead of the neurons in the same column. Hence, the total input to neuron $(g, i)$ can be applied to these two equations and obtained as

$$
\Lambda_{g, i}=-\beta \sum_{j=1}^{m} V_{g, j}-\frac{\alpha}{2} d_{g, j}
$$

The proposed algorithm was found to be convergent during network evolutions.

\section{Estimation of Maneuvering Targets with an Adaptive Filter}

In real-world applications, either maneuvering or non-maneuvering targets must be tracked simultaneously with mobile sensor data association tracking techniques. On the basis of the concept of wireless sensor network equipped with different sensing hardware for distinctly specified applications, a viable method for human interfacing with the environment can be provided. Therefore, if target maneuvers occur, then the maneuver-detection and acceleration-estimation algorithm should be applied to modify the parameters of the tracking filter. The developed methods allow the maneuvers to be tracked without diverging or severely distorting the estimate. To complete such an adaptive procedure, the Kalman filter equations are modified in this subsection. Specifically, certain parameters must be changed for fitting the data association and matching the CHNN algorithm. Now, the measurement innovation, $\rho(k)$, and the innovative covariance matrix, $S(k)$, of the Kalman filter should be modified as

$$
\rho(k)=Y(k)-H(k) \widehat{X}(k \mid k-1)
$$

and

$$
S(k)=\delta(k)-R(k)
$$

, respectively, where $\delta(k)=H(k) P(k \mid k-1) H^{T}(k)$. Furthermore, two hypotheses, $H_{0}$ and $H_{1}$, corresponding to the assumptions that the system behavior is normal and the target is moving 
with the maneuvers, are assumed as generating events. The detection criterion can be defined as

$$
f(k)=\sum_{j=k-n+1}^{k} \rho^{T}(j) S^{-1}(j) \rho(j){\stackrel{c}{H_{1}}}_{<}^{>}
$$

where $f(k)$ is a Chi-squared random variable with $n \times m$ degrees of freedom, where $n$ is the residual window size and $m$ denotes the dimension of the measurement vector, since the noises $V(k)$ and $W(k)$ are assumed to be zero mean Gaussian white noises. In (16) the criterion $\zeta$ can be chosen from standard Chi-squared tables [24]. Moreover, now consider a situation wherein the target initiates and suffers from a severe maneuvering condition. In this case the proposed algorithm will implement the detection and the estimation for the situation on the basis of certain statistical calculations. In this situation, another important algorithm is also applied to increase the estimation accuracy. In such an algorithm, for the $i-t h$ component of a vector, on the basis of the detection results, the testing condition with the components which have jumps are expressed as

$$
\left|\rho_{i}(k)\right| \leq\left|D \sqrt{S_{i i}(k)}\right|
$$

where $D$ represents the rejected innovation, being a constant related to the Gaussian pdf. The variance of the rejected innovation can be calculated as

$$
\operatorname{var}\left(D^{2}\right)=\rho_{i}^{2}(k)\left\{a_{i}(k) \delta_{i i}(k)+R_{i i}(k)\right\}^{-1}
$$

where $\operatorname{var}(\cdot)$ is variance operator; the parameter $a_{i}(k)$ can be computed as

$$
a_{i}(k)=\frac{\left[\rho_{i i}(k) / D\right]^{2}-R_{i i}(k)}{R_{i i}(k)}
$$

Once the parameters have been modified and determined by Eq. 18 and 19, the innovative values $\rho(k)$ at time $k$ can exist on the boundaries of the acceptable region defined by 17 . Thus, to keep the target on track, the covariance of the prediction error $P(k \mid k-1)$ is modified to $\left[a_{m}(k) \cdot P(k \mid k-1)\right]$, where $a_{m}(k)$ is the largest value of all the $a_{i}(k)$ shown in 19. By virtue of the data association algorithm, not only is a better performance obtained, but the tracking filter also has faster responses in server-maneuvering situations. However, under such a procedure, i.e., after the approach is adopted, the Kalman gain increases gradually, after which the tracking performance of the WSS will become more stable and efficient.

\section{Simulation Results and Discussion}

Developing simulation programs (using Matlab ) by virtue of the proposed DFA is implemented in this subsection. The developed DFA associating with CHNN technique is first validated in an environment wherein three targets are tracked in WSN deployments. Next the DFA is also applied to track five targets, including two non-maneuvering and three maneuvering ones. The initial conditions for simulating the tracking of two targets are listed in Table I, mentioned here mainly for demonstrating the accuracy and efficiency of the proposed algorithms. The transition matrix $F(k)$ and the noise gain matrix $G(k)$ corresponding to the target for the sampling interval $T$, which is assumed in the simulation to be two seconds, are given by 
$F(k)=\left[\begin{array}{cccc}1 & T & 0 & 0 \\ 0 & 1 & 0 & 0 \\ 0 & 0 & 1 & T \\ 0 & 0 & 0 & 1\end{array}\right]$ and $G(k)=\left[\begin{array}{cc}T^{2} / 2 & 0 \\ T & 0 \\ 0 & T^{2} / 2 \\ 0 & T\end{array}\right]$, respectively. The initial value of the state error covariance is assumed to default as $P(0 \mid 0)=\left[\begin{array}{cccc}10000 & 100 & 0 & 0 \\ 0 & 100 & 100 & 0 \\ 0 & 0 & 10000 & 100 \\ 0 & 0 & 100 & 100\end{array}\right]$. After the assignment of initial conditions is completed, the procedure of simulation for the DFA algorithm is following the steps illustrated in Fig. 3. The results from tracking three targets without and with the DFA calculation are graphed in Fig. 4 and Fig. 5, respectively. In these simulations eighty steps Monte Carlo are implemented; moreover, 10 estimated tracking (measurements) with dataassociation calculation are sampled for reciprocal comparison for accuracy. It is easy to see that the many more matching situations occur in Fig. 5. I.e., all of the tracking paths tightly parallel the true path marked with circles. It should be emphasized that a little difference does exist the paths of the true targets and the results presented in Fig. 4 and Fig. 5, since the tracking is generated with a random function of the software program. Usage of random-number generators for the measurement of noise and clutter points is illustrated in the simulation. Furthermore, a Kalman filter is utilized to recursively estimate the state vector $\widehat{X}(k \mid k)$. On the basis of each hypothesis formulated from the measurement data received, the corresponding correlations can be promptly calculated. Hence, the position errors caused by the use and non-use of DFA calculation and without DFA calculation are plotted in Fig. 6, and Fig. 7, respectively. It is reasonable to state that the larger position error, with an order of ten thousands, is generated by the results obtained prior to DFA calculation; whereas, a smaller position error, in an approximate order of hundreds, occurs in the case after DFA calculation. A case with all the noise uncorrelated is assumed in the simulation. Thus the values $\alpha=1$ and $\beta=r+1$ are chosen in the simulation for five targets and ten to thirty measurements, where $r$ is the radians of the gate. After eighty Monte Carlo iterations the simulation results from tracking the five targets, two non-maneuvering (Target 1 and Target 2) and three maneuvering (Target 3 to Target 5), are graphed in Fig. 8. The results of the tracking position errors after DFA calculation are plotted in Fig. 9. According to the simulation results based on several different situations, we know that the performance of the proposed algorithm, DFA on CHNN-based, is quite well. The proposed CHNN-based DFA algorithm might have a hardware complexity (number of neurons) in direct linear proportion to the number of tracked targets and the deployed mobile sensors in WSSs. Moreover, tracking for targets with mobile sensors by using the DFA algorithm in WSNs is constrained by the requirement for training the CHNN. However, this CHNN can be thoroughly implemented in analog VLSI technology with currently existing methods. Therefore, the authors believe that, overall, since a CHNN is more appropriate for a neural network based in multi-mobile sensor tracking environments, one may expect to see many such applications in WSS constructions in the near future. Furthermore, although in this type of environment the size of the optimization problem requiring much attention is considerably larger, the proposed DFA algorithm performs quite well, as in the simulation illustration. This is probably due to a sparse assumption of fewer tracked targets. However, the scale of the CHNN that would be required for the implementation of a practical mobile sensor might become a problem when the number of sensors and the measurements become very large. A current issue for development is reduction of the scale of the quadratic optimization problem that the CHNN must solve, so that available analog CHNN IC (integrated circuit) implementation can be used to build practical mobile sensors. Besides, the trends for implementing the WSS in large scale network are 
generally to distribute the fused-data in some small area networks separately. The DFA based on CHNN proposed in this paper, thus, can be implemented in the various mobile environments both in large scale and small scale networks. Finally, since the amount of energy consumed by a mobile sensor during the processing of the DFA is large, such consumption is another important issue. Recently, several methods have been proposed for investigating energy-awareness problems in the mobile sensors in WSS [25]. The authors are currently working on developing a method for decreasing energy consumption by a mobile sensor so that the lifetime of sensors in WSS can be increased.

Table 1: Initial conditions of two targets

\begin{tabular}{|c|c|c|c|c|}
\hline & $x(m)$ & $\dot{x}(m / s)$ & $y(m)$ & $\dot{y}(m / s)$ \\
\hline Target 1 & 1500 & 400 & 3500 & 560 \\
\hline Target 2 & 1000 & 600 & 4000 & 440 \\
\hline
\end{tabular}

Table 2: Initial conditions of five targets

\begin{tabular}{|c|c|c|c|c|}
\hline & $x(m)$ & $\dot{x}(m / s)$ & $y(m)$ & $\dot{y}(m / s)$ \\
\hline Target 1 & 100 & 400 & 3500 & 560 \\
\hline Target 2 & 1000 & 600 & 400 & 440 \\
\hline Target 3 & 0 & 550 & 8000 & 80 \\
\hline Target 4 & 20 & 540 & 9400 & -100 \\
\hline Target 5 & 0 & 0 & 12500 & -70 \\
\hline
\end{tabular}

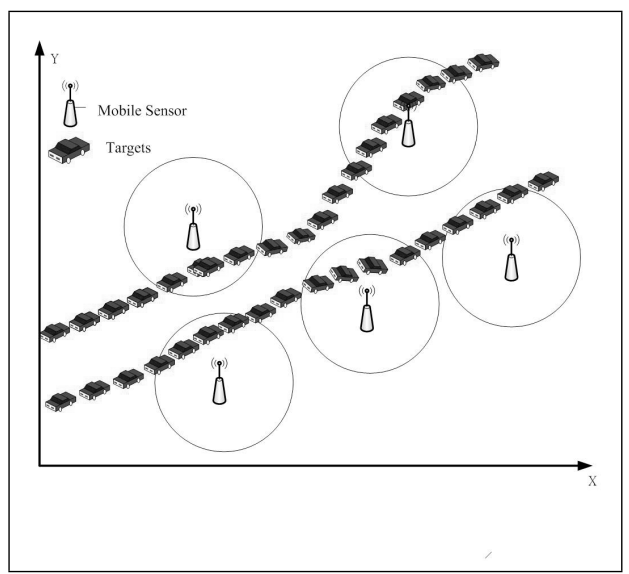

Figure 1: Deployment with two targets and five mobile sensor nodes, with sensing areas covered in circles. 


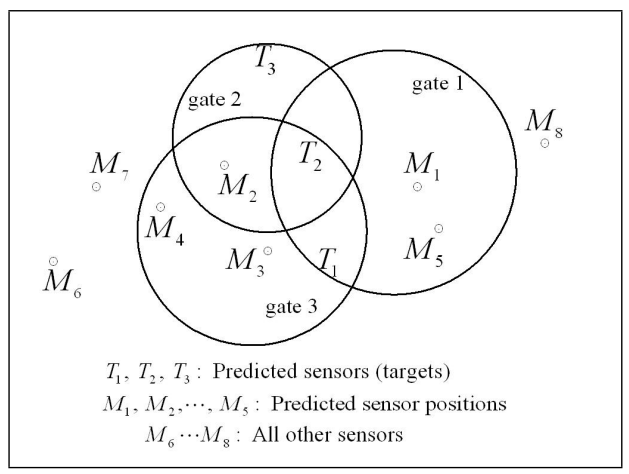

Figure 2: Relationships between measurements and predicted targets, based on gating technique.

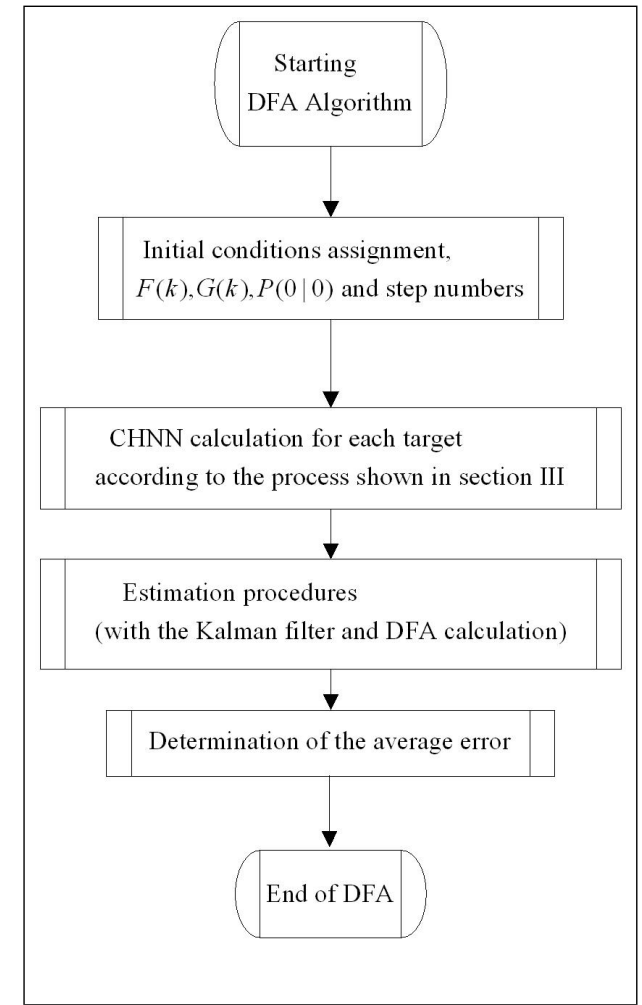

Figure 3: The procedure of simulation for the DFA algorithm.

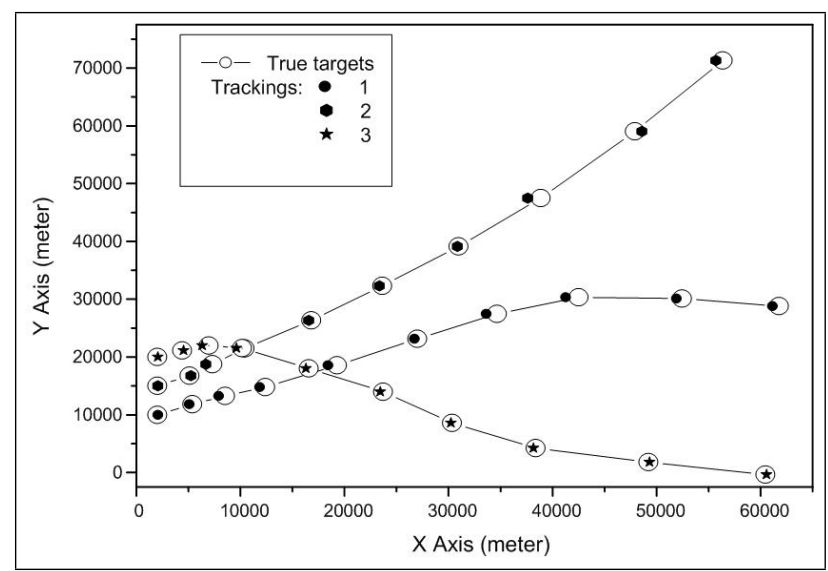

Figure 4: Data association results for tracking three targets prior to DFA calculation. 


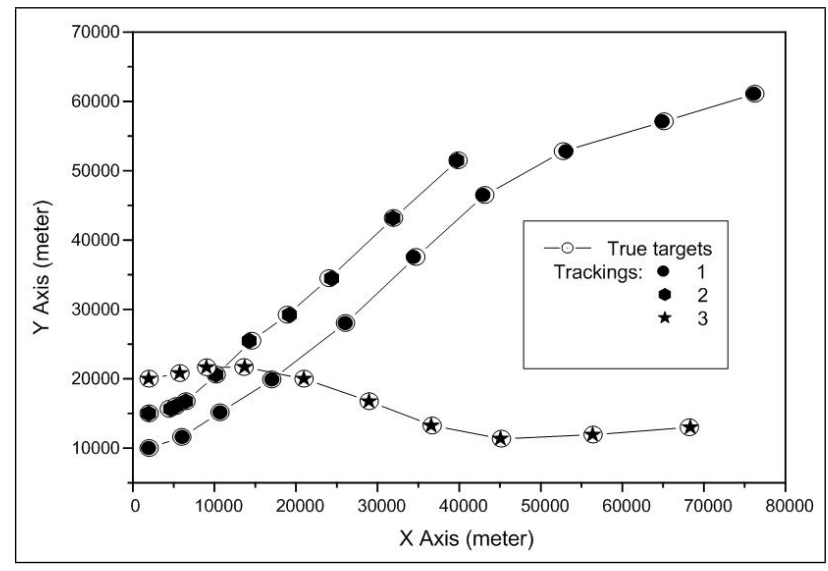

Figure 5: Data association results for tracking three targets after DFA calculation

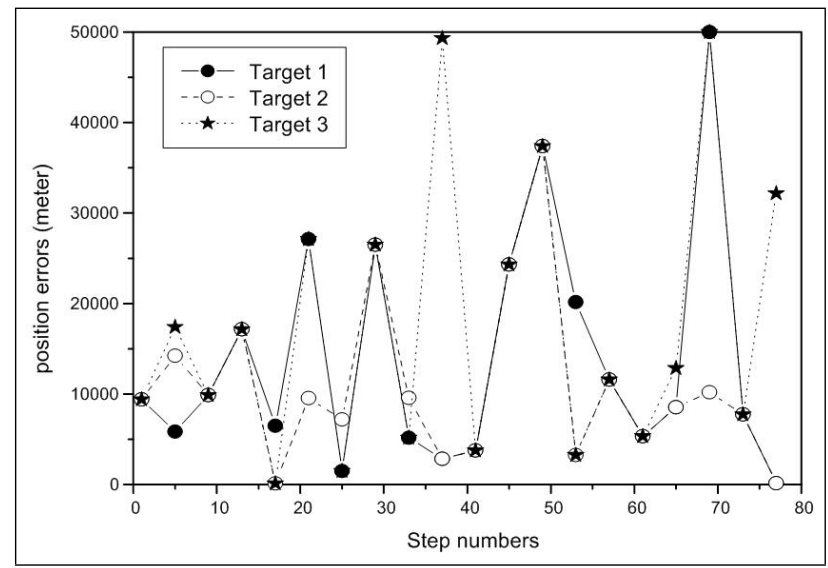

Figure 6: Position errors in tracking three targets prior to DFA calculation.

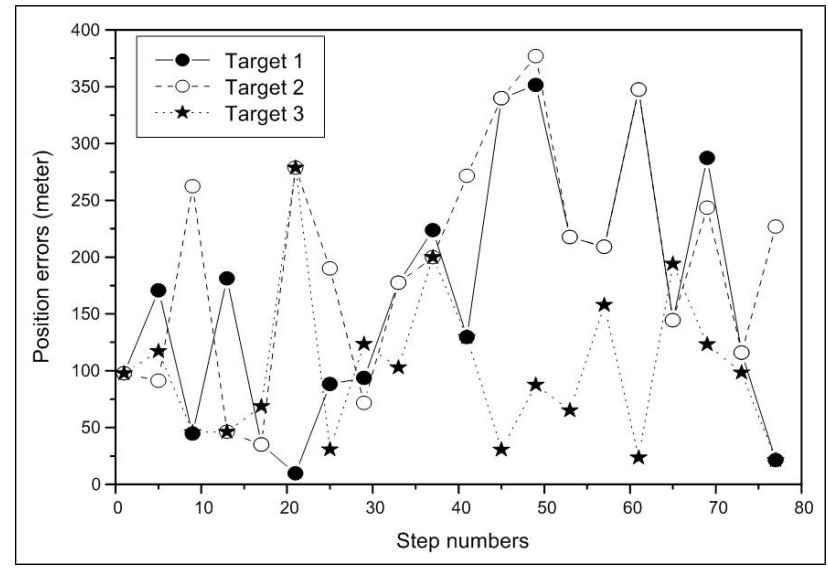

Figure 7: Position errors in tracking three targets after DFA calculation. 


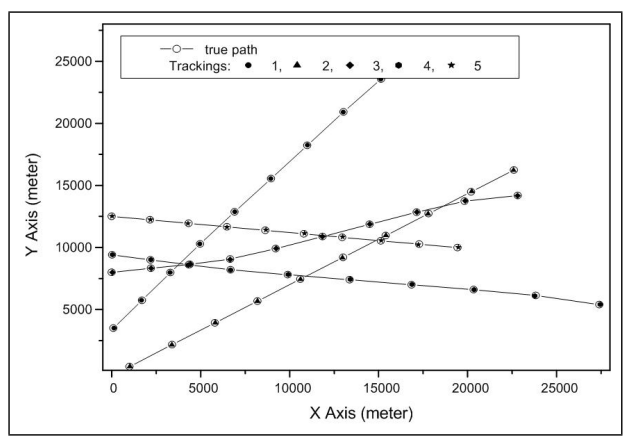

Figure 8: Simulations of tracking of five targets (using CHNN algorithm).

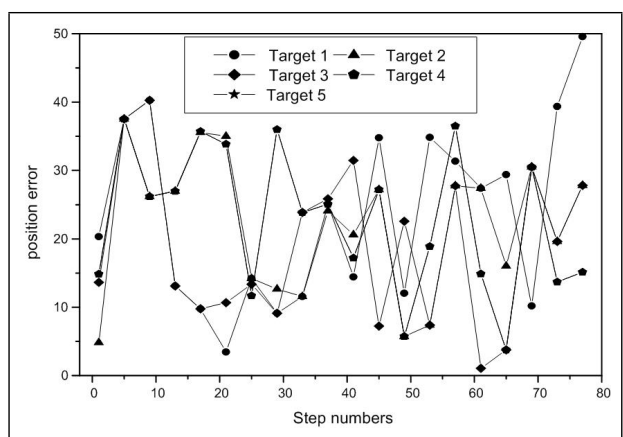

Figure 9: Position errors in tracking six targets after DFA calculation (using CHNN technique).

\section{Conclusions}

An innovative DFA algorithm based on CHNN techniques for tracking multiple targets with mobile sensors over WSS environments has been proposed in this report. This tracking technique has been investigated for its advantages in choosing an optimal correlation between mobile sensor measurements and existing target tracks. Because of the application of the CHNN technique, it was discovered that the system is relieved of the burden of determining the proper weighting factors as in a traditional HNN; therefore, the network can always achieve a rational solution. Moreover, an adaptive procedure for tracking maneuvering targets is also employed in this algorithm via stochastic process obtained by the DFA. On the basis of the simulation results obtained in this study, it can be claimed that this DFA algorithm is capable of obtaining the optimal correlations between true targets and mobile sensor measurements in WSS scenarios. Finally, the approach developed in this research has demonstrated not only stable performance for tracking procedures but definitely also excellent efficiency when tracking both constant velocity and maneuvering targets.

\section{Acknowledgement}

The author expresses appreciation to Dr. C. Rutledge, Department of English, Dayeh University, for her editorial assistance. The authors also would like to thank the anonymous reviewers for many useful comments which help to improve the quality and readability of this paper. 


\section{Bibliography}

[1] F. Zhao, L. Guibas, Wireless Sensor Networks: An Information Processing Approach. Elsevier Pte. Ltd., Singapore, 2004.

[2] M. Cetin, Lei Chen, Fisher, J. W., III, Ihler, A. T., Moses, R. L., Wainwright, M. J. Willsky, A. S., Distributed Fusion in Sensor Networks. IEEE Signal Processing Magazine, vol. 23, issue 4, pp. 42-55, 2006.

[3] J. Miguez, A. Artes-Rodriguez, Monte Carlo Algorithms for Tracking a Maneuvering Target Using a Network of Mobile Sensors. Proc. 1st IEEE Int. Workshop Computational Advances in Multi-Sensor Adaptive Processing, Puerto Vallarta, Mexico, vol. 1, pp. 89-92, 2005.

[4] K. C. Chang, C. Y. Chong, Y. Bar-Shalom, Joint Probabilistic Data and Association Distributed Sensor Networks. IEEE Transactions on Automatic Control, vol. AC-31, pp. 889897, 1986.

[5] N. Okello, B. Ristic, Maximum Likelihood Registration for Multiple Dissimilar Sensors. IEEE Transactions on Aerospace Electronic Systems, vol. 39, issue 3, pp. 1074-1083, 2003.

[6] Y. Bar-Shalom, T. E. Fortmann, Tracking and Data Association. Academic Press, Inc., 1989.

[7] S.S. Blackman, Multiple Hypothesis Tracking for Multiple Target Tracking. IEEE Aerospace Electronic Systems, vol. 19, issue 1, pp. 5-18, 2004.

[8] Y.N. Chung, J. I. -Z. Chen, Applying Both Kinematic and Attribute Information for a Target Tracking Algorithm. Journal of Control Systems and Technology, pp. 203-209, 1997.

[9] C. Hue, Le Cadre., J. -P., P. Perez, Sequential Monte Carlo Methods for Multiple Target Tracking and Data Fusion. IEEE Transactions on Signal Processing, vol. 50, issue 2, pp. 309-325, 2002.

[10] D. Sengupta, R.A. Iltis, Neural Solution to the Multitarget Tracking Data Association Problem. IEEE Aerospace Electronic Systems, vol. 25, issue 1, pp. 96-108, 1989.

[11] L. Chin, Application of Neural Networks in Target Tracking Data Fusion. IEEE Aerospace Electronic Systems, vol. 30, issue 1, pp. 281-287, 1994.

[12] C. Y. Chang, P. C. Chung, Medical Image Segmentation Using a Contextual-constraint Based Hopfield Neural Cube. Image and Vision Computing, pp. 669-678, 2001.

[13] E. Soujeri, H. Bilgekul, Hopfield Multiuser Detection of Asynchronous MC-CDMA Signals in Multipath Fading Channels. IEEE Communications Letters, vol. 6, issue 4, pp. 147-149, 2002 .

[14] B. Zhou, N. K. Bose, A Comprehensive Analysis of Neural Solution to the Multitarget Tracking Data Association Problem. IEEE Aerospace Electronic Systems, vol. 29, issue 1, pp.260-263, 1993.

[15] X. Wang, A. Jiang, S. Wang, Mobile Agent Based Moving Target Methods in Wireless Sensor Networks. Proc. IEEE Int. Symp. Commun. and Info. Tech., Beijing, China, vol. 1, pp. 22-26, 2005. 
[16] Q. Liang, D. F. Yuan, Y. Wang, R. H. Zhang, A New Sensor Antenna-array Selecting Method in Wireless Sensor Networks. In Proceeding Int. Conf. on Communications, Circuits and Systems, Guilin, China, vol. 3, pp. 1523-1526, 2006.

[17] S. Y. Kung, Digital Neural Networks. PTR Prentice Hall, Englewood Cliffs, New Jersey, 1993.

[18] X. Wang, S. Wang, D. Bi, Dynamic Sensor Node Selection Strategy for Wireless Sensor Networks. In Proceeding IEEE Int. Symp. Commun. and Info. Tech., Darling Harbour, Sydney, Australia, vol. 1, pp. 1137-1142, 2007.

[19] T. Semertzidis, K. Dimitropoulos, A. Koutsia, N. Grammalidis, Video Sensor Network for Real-time Traffic Monitoring and Surveillance. IET Intelligent Transport Systems, vol. 4, Issue 2, pp. 103-112, 2010.

[20] Y. -S. Yen, S. Hong, R. -S. Chang, H. -C. Chao, Controlled Deployments for Wireless Sensor Networks. IET Communications, vol. 3, Issue 5, pp. 820-829, 2009.

[21] Y. Liu, N. Xiong, Y. Zhao, A.V. Vasilakos, J. Gao, Y. Jia, Multi-layer Clustering Routing Algorithm for Wireless Vehicular Sensor Networks. IET Communications, vol. 4, Issue 7, pp. 810-816, 2010.

[22] L. Shi, A. Capponi, K. H. Johansson, R. M. Murray, Resource Optimization in a Wireless Sensor Network with Guaranteed Estimator Performance. IET Control Theory Applications, vol. 4, Issue 5, pp. 710-723, 2010.

[23] M. S. Grewal, A. P. Andrew, Kalman Filtering, Theory and Practice-using MATLAB, 2nd ed. John Wiley 6 S Sons, Inc., New York, 2001

[24] A. Papoulis, S. U. Pillai, Probability, Random Variables, and Stochastic Processes. 4th ed. McGraw-Hill, Comp., Inc., New York, 2002

[25] X. Wang, D. Wang, Y. Wang, Agrawal, D. P., A. Mishra, On Data Fusion and Lifetime Constrains in Wireless Sensor Networks. In Proceeding IEEE Int. Communication. Conf., Glasgow, Scotland, vol. 9, pp. 3942-3947, 2007. 